\title{
Valoración de los resultados en salud percibidos y comunicados por los pacientes: ¿necesidad o lujo?
}

\author{
J. SOTO ÁLVAREZ
}

Dpto. de Investigación de Resultados en Salud y Farmacoeconomía. Unidad MédicaPfizer I+D+i-Madrid

Soto Álvarez J. Valoración de los resultados en salud percibidos y comunicados por los pacientes: ¿necesidad o lujo? An Med Interna (Madrid) 2006; 136-138.

En los albores del siglo XXI, en nuestro país los pacientes han dejado de ser meros receptores de la atención sanitaria para convertirse en una pieza clave dentro del sistema sanitario, de tal manera que cada vez más la política sanitaria y su estrategia de implantación se planifica y ejecuta hacía y en torno a las necesidades e intereses de los pacientes.

A este hecho ha contribuido de manera importante la mejor formación sanitaria y el mayor nivel cultural alcanzado por la sociedad española en los últimos años, así como la llegada de las nuevas tecnologías de la información (especialmente, Internet), lo que ha hecho que los pacientes estén más y mejor formados e informados. Por otra parte, el profesional sanitario cada vez es más consciente de la necesidad de que el paciente disponga de mayor información sobre su patología y las posibles terapias existentes para la misma, y que debe ser escuchado y estar implicado y participar en la toma de decisiones que afectan a su salud y bienestar (1-3).

Fruto de esta nueva corriente que trata de implicar de manera más directa a los pacientes en todas los temas que se refieren y tienen como objetivo el cuidado sanitario y la toma de decisiones en este ámbito, en Mayo de 2003 se elaboro la Declaración de Barcelona de las Asociaciones de Pacientes (4), embrión del actual Foro Español de Pacientes (5), el cuál proclama la necesidad de una información de calidad en temas sanitarios a los pacientes, la conveniencia de que las decisiones en atención sanitaria se centren y giren en torno a éstos y su participación activa a la hora de decidir la política sanitaria del país.

De manera adicional, toda esta nueva filosofía de una atención sanitaria centrada en el paciente también ha tenido su repercusión en nuestro sistema jurídico, y de hecho, tanto la Ley de Cohesión y Calidad del Sistema Nacional de Salud (6), como la Ley de Autonomía del Paciente (7) establecen el derecho del paciente a conocer toda la información disponible relativa a su salud, así como su participación activa no sólo en lo que se refiere a las decisiones clínicas (a través de los foros de dialogo social), sino también en el establecimiento y fijación de políticas sanitarias.

Parece claro, por lo tanto, que el paciente va a ser una de las piezas claves en el futuro a la hora de diseñar y establecer estrategias y planes en los Sistemas Sanitarios de los países industrializados, y que cada vez su voz va a ser tenida más en cuenta en el proceso de toma de decisiones, tanto por la clase médica como por la esfera política. Este hecho es de una importancia capital, ya que cualquier sujeto en nuestra sociedad ha sido, es o será paciente de manera irremediable, y cuando esto suceda deberá tener derecho a ser informado correctamente de su dolencia, a que su opinión sea tenida en cuenta a la hora de tomar decisiones sobre su propia enfermedad y a ser consultado para la elaboración e implantación de políticas y actuaciones en materia sanitaria.

En este contexto, cada vez va a ser más primordial poder conocer cómo percibe y le afecta al paciente de manera subjetiva la enfermedad que padece (y sus posibles complicaciones) y las terapias administradas para su tratamiento, esto es, disponer de información desde la perspectiva del paciente de cómo la enfermedad y su tratamiento afectan a su vida cotidiana y, además, esta información debería ser comunicada adecuadamente a los profesionales de la salud y otros decisores sanitarios.

La forma de lograr estos dos objetivos de manera valida y fiable es evaluando los resultados en salud percibidos y comunicados por el paciente de una manera objetiva, precisa y con rigor científico. Estos datos, denominados patient-reported

Trabajo aceptado: 11 de enero de 2006

Correspondencia: Javier Soto Álvarez. Dpto. de Investigación de Resultados en Salud Unidad Médica Pfizer I+D+i. Avda. de Europa, 20-B. 28108 Alcobendas (Madrid). e-mail: javier.soto.alvarez@pfizer.com 
outcomes (PRO) en los países anglosajones, representan cualquier resultado basado en la percepción y comunicación del paciente sobre su enfermedad y el tratamiento recibido, así como las repercusiones de ambas en su manera de vivir y relacionarse (8).

Los resultados en salud percibidos y comunicados por el paciente engloban un conjunto de sensaciones y vivencias subjetivas y valoraciones efectuadas por el mismo, las cuáles van desde la medición de una sola dimensión o característica de la enfermedad (intensidad de un síntoma clínico, evaluación global de un tratamiento, mejoría clínica global, etc.) hasta valoraciones de diferentes dimensiones de la patología a través de cuestionarios estructurados, tales como sintomatología global de la enfermedad, situación funcional, satisfacción con el tratamiento recibido, satisfacción con la vida, sensación de bienestar, calidad de vida relacionada con la salud y cumplimiento terapéutico (9-11).

Este tipo de resultados representa un indicador esencial y único para poder valorar cómo el paciente siente y vive la enfermedad y su tratamiento, y en muchas ocasiones, va a reflejar la efectividad del tratamiento administrado y la evolución de la propia enfermedad de manera más fidedigna que otros marcadores empleados habitualmente para tal cometido, como analíticas, pruebas de función de distintos órganos, empleo de técnicas radiológicas, etc.

En muchas patologías, tales como las enfermedades funcionales del tracto gastrointestinal (dispepsia, colón irritable, etc.), la disfunción sexual, el dolor o distintas alteraciones psiquiátricas (depresión, ansiedad, etc.), por poner algunos ejemplos, el manejo, control y monitorización de la afección por parte del profesional sanitario se va a basar, primordialmente, en la percepción y comunicación de los síntomas por parte de los pacientes y cómo éstos impactan en su funcionamiento en la vida diaria y en su sensación de bienestar.

En otro tipo de enfermedades donde no existen marcadores físicos o fisiológicos de su actividad, los resultados en salud que nos relatan los propios pacientes van a ser los parámetros de elección para evaluar la progresión y la afectación del paciente por la enfermedad que padece.

Por estos motivos, cada día es más importante conocer aspectos distintos de los empleados clásicamente para evaluar la afectación del pacientes por la enfermedad que padece (valores anómalos de pruebas analíticas y aparición y/o empeoramiento de síntomas objetivos para el médico) y para valorar el cociente beneficio/riesgo de los tratamiento empleados (eficacia, tolerabilidad y seguridad). Estos nuevos atributos tendrán que reflejar cómo los pacientes perciben y sienten que su dolencia y el tratamiento administrado les esta afectando en su vida cotidiana. De hecho, este tipo de resultados en salud percibidos y comunicados por los pacientes ya son incluidos en las fichas técnicas de muchos medicamentos, como un valor terapéutico añadido de los mismos (12), y cada día se incluyen más mediciones de estos datos en los protocolos de los ensayos clínicos y diseños observacionales efectuados con medicamentos (13-15).

El sistema sanitario es uno de los pilares básicos del estado de bienestar logrado por la sociedad en nuestro país. Todos los ciudadanos, co-propietarios y financiadores de nuestro sistema nacional de salud (SNS), utilizamos sus servicios a lo largo de nuestra existencia, en unos casos de manera preventiva y en otros curativa, pero todos vamos a ser pacientes antes o después. Por lo tanto, es esencial que la opinión y voz del paciente sea tenida en cuenta en los foros donde se discuten y abordan los planes de actuación del SNS.

Por otra parte, el paciente del futuro va a ser diferente en muchos aspectos al paciente actual, ya que va a demandar una mayor información y va a exigir un mejor nivel de servicios junto con una elevada calidad asistencial por parte del SNS y de todo el personal sanitario, lo que exigirá un mayor flujo de comunicación bi-direccional entre ambos. Por lo tanto, los profesionales sanitarios van a tener que estar preparados para aceptar el reto que supone atender a un paciente más autónomo y mejor informado y, por lo tanto, les va a ser imprescindible conocer como la enfermedad y su tratamiento está afectando al paciente en su vida rutinaria $(16,17)$.

Conocer y disponer de resultados en salud percibidos y comunicados por los pacientes es en estos momentos una clara necesidad (y no un lujo, como se sigue pensando en algunos reductos), y todos los estamentos del SNS deberían velar por que esto fuera una realidad. Por este motivo, debería incluirse su medición y cuantificación en todos los estudios clínicos que se realicen, incorporando instrumentos y cuestionarios existentes en nuestro país para tal cometido, una vez hayan sido validados y adaptados culturalmente de manera apropiada.

De manera paralela, es necesario profundizar en la manera de utilizar, analizar e interpretar los resultados que nos proporcionan estos cuestionarios, de tal manera que se pueda estandarizar lo que se considera como diferencia clínicamente importante a la hora de evaluar las puntuaciones obtenidas, lo que ayudaría de forma importante a la hora de poder tomar decisiones (18). Además, es importante seguir trabajando para disponer de cuestionarios cada vez más sensibles, fáciles de administrar y de analizar y, sobre todo, que les sean relevantes y útiles en la práctica clínica diaria a los profesionales sanitarios.

En otro orden de cosas, sería conveniente que se crease un instituto de investigación de resultados en salud notificados por los pacientes dentro del proyecto marco del Ministerio de Sanidad de crear institutos sanitarios para potenciar la rápida incorporación de las innovaciones científicas a la práctica clínica, lo que permitiría orientar la investigación del Sistema Sanitario al logro del mayor beneficio para los pacientes.

La potenciación de redes de investigación que trabajen en este campo, la red Iryss (red sobre investigación de resultados en salud y servicios) (19) es un buen ejemplo, y la creación de otras posibles redes, también contribuiría de manera importante a que se dispusiera de resultados en salud percibidos y comunicados por el paciente en distintas patologías y en diferentes subgrupos de pacientes.

Por otra parte, la inclusión de la necesidad de cuantificar y evaluar los resultados en salud percibidos por los pacientes de manera rutinaria dentro del SNS en las futuras Leyes de Garantía y Uso Racional del Medicamento y Productos Sanitarios y de Investigación Biomédica, en las cuáles está trabajando actualmente el Ministerio de Sanidad y Consumo, y qué se prevé que puedan estar promulgadas antes de final del año en curso, ayudaría enormemente a que la investigación en esta área fuera una realidad.

La sanidad es uno de los bienes públicos con un crecimiento más dinámico y qué va a necesitar más recursos en el futuro, los cuáles van a ser imprescindibles para dar solución 
a las mayores demandas por parte de los ciudadanos, cada vez más informados y con una mayor exigencia de mejores servicios. Conocer las percepciones subjetivas de los pacientes en relación a su enfermedad y los tratamientos administrados, va a ser de gran utilidad para logara disponer de un SNS más orientado al paciente y de mayor calidad asistencial, lo que redundará en una mejora global de la salud y el bienestar de los ciudadanos, fin último de cualquier Sistema Sanitario.

\section{Bibliografía}

1. Nebot C, Baqués T, Crespo A, Valverde I, Canela-Soler J. La opinión de los usuarios como oportunidad de mejora en atención primaria. Aten Primaria 2005; 35: 290-294

2. Alonso-Coello P, Solà I, García JM. Valores y preferencias de los pacientes: asignatura pendiente. Aten Primaria 2005; 35: 379

3. Wright JG. Evaluating the outcome of treatment: shouldn't we be asking patients if they are better? J Clin Epidemiol 2000; 53: 549-553

4. Declaración de Barcelona de las Asociaciones de Pacientes 2003 (www.fbjoseplaporte.org/docs/Declaracion_Barcelona_2003.pdf) (consultado 19/Septiembre/2005)

5. Manifiesto del Foro Español de Pacientes (www.fbjoseplaporte.org/ docs/manifiesto,pdf) (consultado 19/Septiembre/2005)

6. Ley $16 / 2003$, de 28 de mayo, de cohesión y calidad del Sistema Nacional de Salud. BOE de 29 mayo 2003.

7. Ley $41 / 2002$, de 14 de noviembre, básica reguladora de la autonomía del paciente y de derechos y obligaciones en materia de información y documentación clínica. BOE 15 noviembre 2002.

8. Doward LC, McKenna SP. Defining patient-reported aoutcomes. Value Health 2004; 7 (Suppl 1): S4-S8

9. Revicki D. Consistent patient-reported outcomes. Value Health 2002; 5: 295-296

10. Casas Anguita J, Repullo Labrador JR, Pereira Candell J. Medidas de calidad de vida relacionada con la salud. Conceptos básicos, construcción y adaptación cultural. Med Clin (Barc) 2001; 116: 789-796.

11. Shikiar R, Rentz AM. Satisfaction with medication: an overview of conceptual, methodological, and regulatory issues. Value Health 2004; 2: 204-215.

12. Willke RJ, Burke LB, Erickson P. Measuring treatment impact: a review of patient-reported outcomes and other efficacy endpoints in approved product labels. Control Clin Trials 2004; 25: 535-552.

13. Acquadro C, Berzon R, Dubois D, Leidy NK, Marquis P, Revicki D, et al. for the PRO Harmonization Group. Incorporating the patient's perspective into drug development and communication: an ad hoc task force report of the patient-reported outcomes (PRO) harmonization group meeting at the Food and Drug Administration, February 16, 2001. Value Health 2003: 6: 522-531.

14. Greenhalgh J, Long AF, Flynn R. The use of patient reported outcome measures in routine clinical practice: lack of impact or lack of theory? Soc Sci Med 2005; 60: 833-843.

15. Wiklund I. Assessment of patient-reported outcomes in clinical trials: the example of health-related quality of life. Fundam Clin Pharmacol 2004; 18: 351-363.

16. Barce Fernández I, Parejo Miguez R, Gutiérrez Martín P, Fernández Alarcón F, Alejandro Lázaro G, López de castro F. La información al paciente y su participación en la toma de decisiones clínicas. Aten Primaria 2004; 33: 641-644.

17. Ruiz Moral R, Prados Torres D, Cabrera Rodríguez JM. La voz de los pacientes: mejorando el entendimiento entre médicos y pacientes. Aten Primaria 2004; 34: 277-278

18. Guyatt GH, Osoba D, Wu AW, Wyrwich KW, Norman GR, and the clinical significance consensus meeting group. Methods to explain the clinical significance of health status measures. Mayo Clin Proc 2002; 77 : 371-383

19. Red sobre Investigación de Resultados en Salud y Servicios (www.rediryss.net/pub/units) (consultado 19 de Septiembre 2005). 